\title{
A Swine Model to Analyze Arterial Structural Changes Induced by Mechanical Thrombectomy
}

\author{
I. Yuki, I. Kan, A. Golshan, J. Sohn, Y. Murayama, H.V. Vinters, and F. Viñuela
}

\begin{abstract}
SUMMARY: We report a novel swine model that allows direct visualization of cervical arteries undergoing mechanical thrombectomy. The model also facilitates evaluation of histologic changes observed in the arteries after treatment. Swine superficial cervical arteries, which are similar in size and branching pattern to the human middle cerebral artery, were surgically exposed, occluded with experimental thrombus, and subsequently treated with the Merci clot retriever device. Angiographic and histologic assessment were performed.
\end{abstract}

ABBREVIATIONS: $\mathrm{Cl}=$ confidence interval; $\mathrm{SCA}=$ superficial cervical artery

$\mathbf{R}$ ecent studies have reported the procedure-related complications of mechanical thrombectomy performed in patients with acute stroke. ${ }^{1-3}$ However, little is known about the exact mechanism of arterial injury in these procedures, partly due to limited angiographic/histologic data from the treated arteries. The purpose of this study was to introduce a new animal model for evaluating arterial structural changes induced by a mechanical thrombectomy device, by using the SCA in swine.

\section{TECHNIQUE}

\section{Angiographic Review of the Superficial Cervical Artery in Swine}

All animal experiments were conducted in accordance with policies established by the University of California, Los Angeles chancellor's animal research committee. Swine are known to have well-developed SCAs that originate directly from the thyrocervical trunk. ${ }^{4,5}$ In the first phase of this study, previously obtained diagnostic angiograms of 40 swine (20 Yorkshire pigs and $20 \mathrm{Yu}-$ catan miniature pigs) were randomly selected and reviewed with particular attention to the anatomy of the thyrocervical trunk and

Received April 27, 2011; accepted after revision May 10, 2012.

From the Division of Interventional Neuroradiology (I.Y., I.K., A.G., F.V.), Department of Radiological Sciences; Division of Laboratory Animal Medicine (J.S.); and Section of Neuropathology and Departments of Pathology and Laboratory Medicine and Neurology (H.V.V.), David Geffen School of Medicine, University of California, Los Angeles, Los Angeles, California; and Department of the Neurosurgery (Y.M.), Jikei University of Medicine, Tokyo, Japan.

H.V.V. is supported in part by the Daljit S. and Elaine Sarkaria Chair in Diagnostic Medicine and SPOTRIAS grant IP50NS044378.

Please address correspondence to Ichiro Yuki, MD, Division of Interventional Neuroradiology, UCLA Medical Center and David Geffen School of Medicine at UCLA, 10833 Le Conte Ave, Los Angeles, CA 90095-1721; e-mail: iyuki@mednet.ucla.edu

http://dx.doi.org/10.3174/ajnr.A3221 the SCA. All 40 angiograms showed well-developed bilateral SCAs (Fig 1). The SCA was seen as a large straight branch projecting superolaterally from the ipsilateral thyrocervical trunk. The mean diameter of the SCA was $2.8 \mathrm{~mm}(95 \% \mathrm{CI}, 2.6-3.0 \mathrm{~mm})$ at its origin and $2.1 \mathrm{~mm}(95 \% \mathrm{CI}, 1.9-2.4 \mathrm{~mm})$ near its bifurcation. The mean diameter of the branches distal to its bifurcation was $1.56 \mathrm{~mm}$ (95\% CI, 1.48-1.64 mm). Given that the outer diameter of the proximal middle cerebral artery in humans was $2.41 \pm 0.41$ $\mathrm{mm}^{6}$ in 1 study and $3.71 \mathrm{~mm}$ (range, $2.74-4.92 \mathrm{~mm}$ ) in another, ${ }^{7}$ the size measurements of the SCA in swine are similar to those of the human middle cerebral artery.

\section{Surgical Exploration of the Superficial Cervical Artery in Yorkshire Pigs}

In the second phase of the study, a total of 6 SCAs in 3 Yorkshire pigs were surgically explored and detailed anatomic orientation was documented. Each animal was intubated, and general anesthesia was maintained by using isoflurane (1\%-3\%) during the procedure. A swine was placed in the supine position, and a $20-\mathrm{cm}$ linear skin incision was made along the course of the sternocleidomastoid muscle. Once the sternocleidomastoid muscle was exposed, connective tissue along its posterior aspect was dissected and a $7-\mathrm{cm}$ segment of the artery, including the origin of thyroid artery and bifurcation of the SCA, was exposed.

\section{ENDOVASCULAR EMBOLIZATION OF THE SUPERFICIAL CERVICAL ARTERY WITH EXPERIMENTAL THROMBUS Occlusion of the Superficial Cervical Artery with Experimental Thrombus}

A total of 6 SCAs in 3 Yorkshire swine were surgically exposed in the same manner as described above. An 8F sheath was placed in the right femoral artery, and subsequently an $8 \mathrm{~F}$ balloon-guiding 


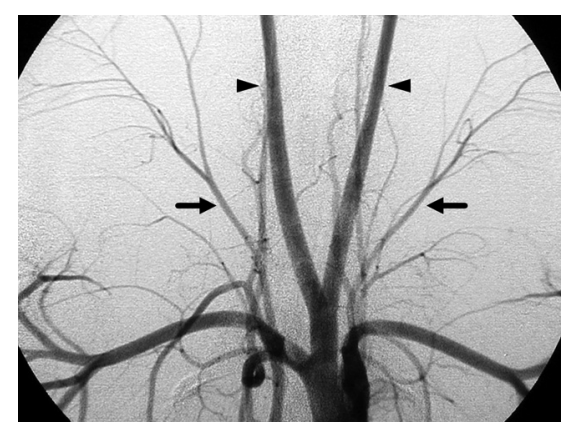

FIG 1. Aortogram of a Yucatan miniature pig (anteroposterior view). Bilateral superficial cervical arteries (arrows) and the main branches of the thyrocervical trunk are seen projecting superolaterally, branching into smaller vessels. Arrowheads indicate bilateral common carotid arteries.

catheter (Merci; Concentric Medical, Mountain View, California) was placed at the origin of the SCA. Under fluoroscopy, 2 SCAs were occluded by injecting experimental thrombus (thrombininduced thrombus ${ }^{8}$ ), and the other 2 were occluded with a differ-
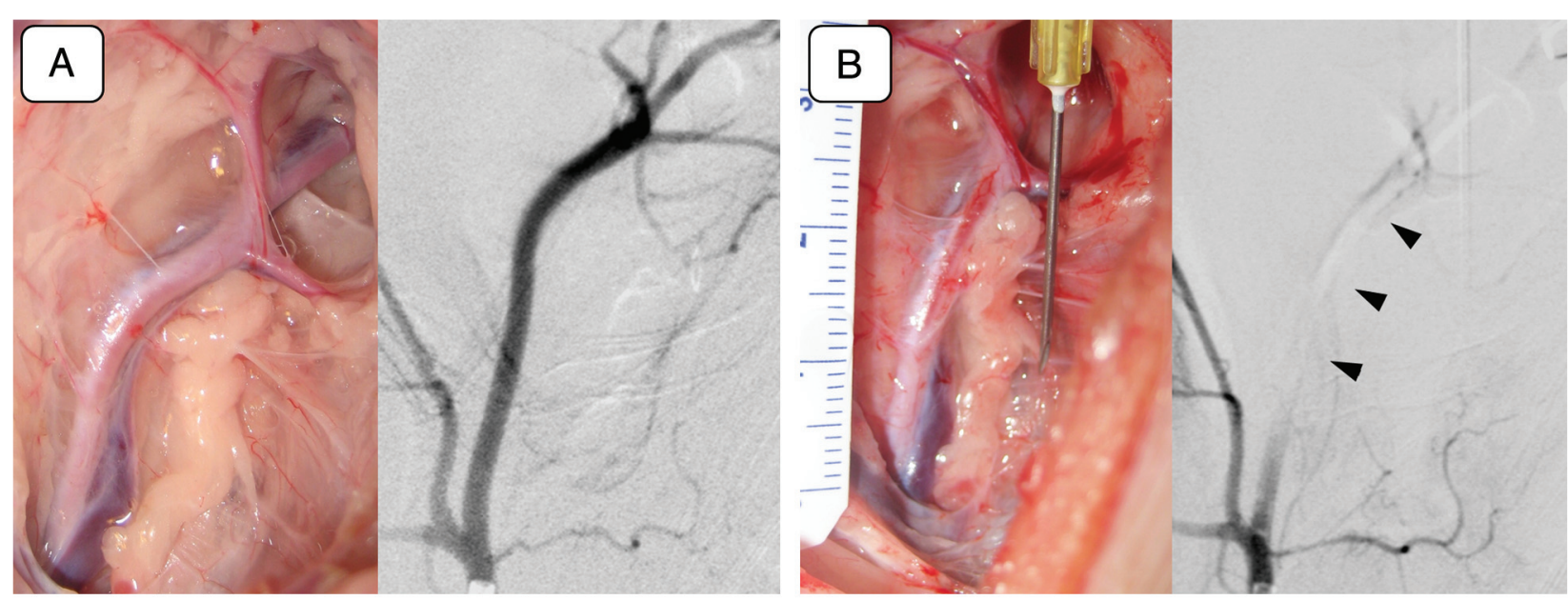

ent type of experimental thrombus (fibrin-rich thrombus ${ }^{9}$ ). The detailed preparation process of each type of thrombus has been published elsewhere. ${ }^{8,9}$ The size of the prepared thrombus was 5 $\mathrm{mm}$ in diameter and $20 \mathrm{~mm}$ in length.Two untreated SCAs were harvested before the injection of clot and were used as controls.

\section{Mechanical Thrombectomy by Using the Merci Clot Retriever System}

Mechanical thrombectomy of the 4 arteries occluded with experimental thrombus was performed with the Merci clot retriever system (Concentric Medical). The use of extracranial arteries in swine to assess the efficacy of mechanical thrombectomy has been well-established and is described elsewhere. ${ }^{8,10}$ Either a V 2.5 Firm or V 3.0 Firm Merci device was selected, depending on the size of the target artery. A total of 11 retrieval attempts were made to achieve the final angiographic results. Three arteries showed recanalization (a Thrombolysis In Myocardial Infarction score of 2) after 2 retrieval attempts, and 1 vessel remained occluded after 5 attempts. The average time to achieve the final angio-

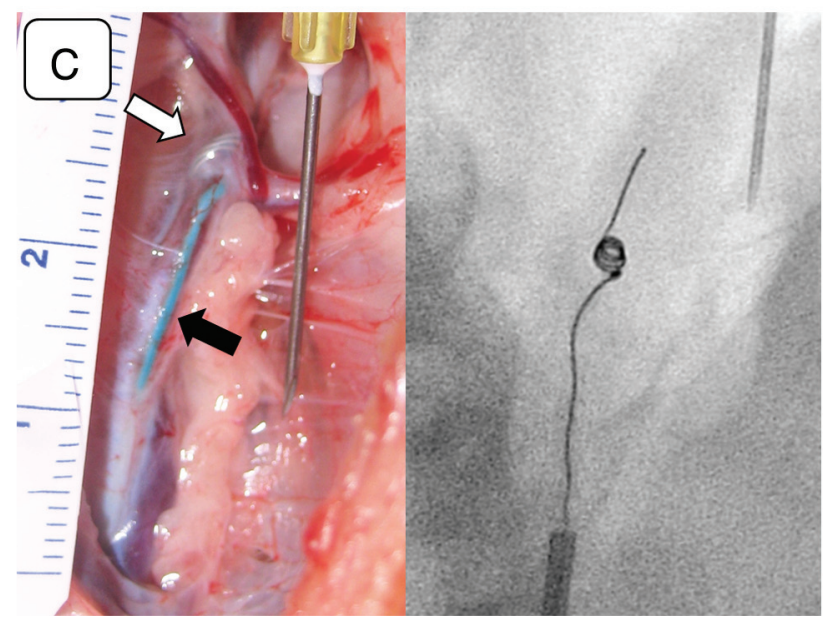

FIG 2. A, Photograph of a surgically exposed left superficial cervical artery (left) and its selective angiogram (right). Note that multiple branches of the superficial cervical artery are seen in both the surgical view and the angiogram. $B$, Intraprocedural photograph (left) and an angiogram (right) of the left superficial cervical artery (postocclusion). The left superficial cervical artery shown in $A$ was occluded with an injected experimental thrombus (left). Pulsation of the artery diminishes significantly after the occlusion, and the artery becomes pale. An angiogram was performed. C, Intraprocedural photograph (left) and an angiogram (right) of the left superficial cervical artery (Merci device deployment). A Merci clot retriever, deployed at the postbifurcation segment of the left superficial cervical artery (open arrow) and a microcatheter (arrow) are seen through the wall of the vessel (left). A fluoroscopic view of the same artery shows the device being deployed and reformed into its original coil shape (right). Behavior of the deployed device can be monitored during the procedure under direct vision. 

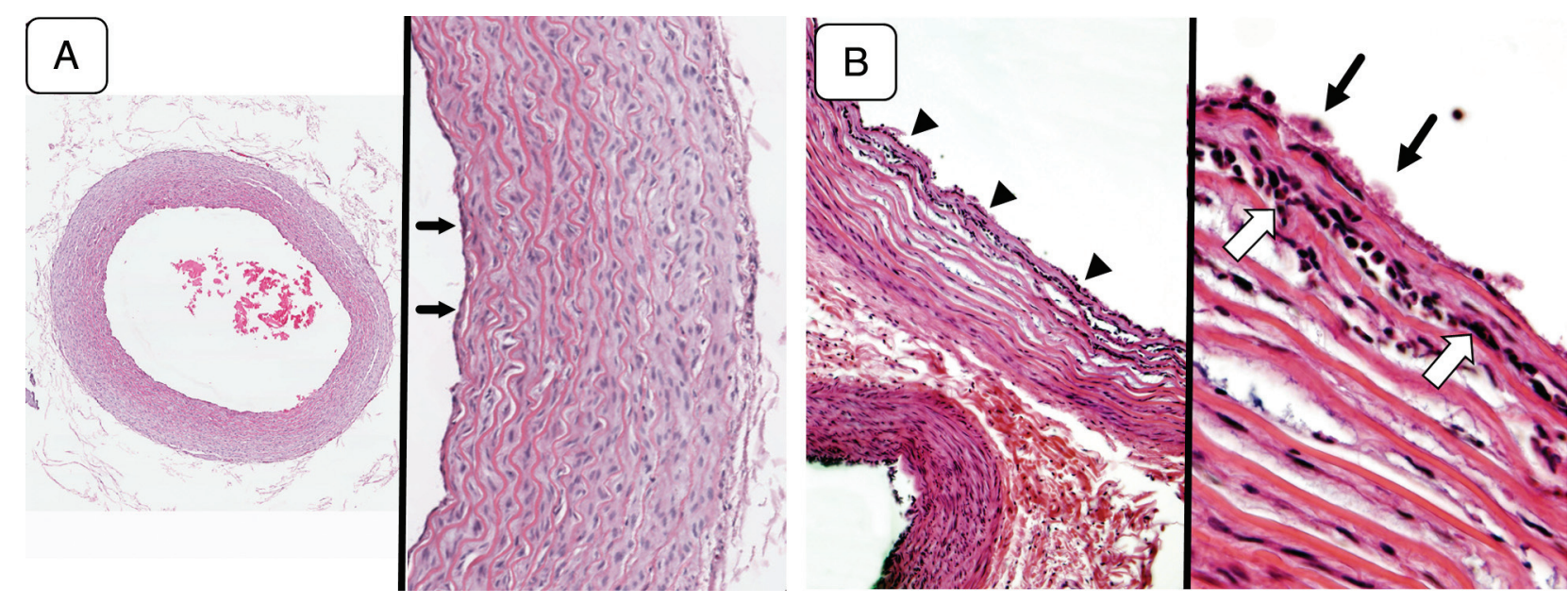

FIG 3. A, Microscopic view of the arterial sample (control group) (hematoxylin-eosin [H\&E] staining). A low-magnification view of the harvested arterial sample (left, original magnification $\times 40$ ) demonstrates well-maintained tissue integrity throughout all layers of the vessel. A magnified view of the same sample (right, original magnification $\times 100$ ) shows the general histologic characteristics of a small artery. The tunica media, which constitutes most of the arterial wall, has up to 10-12 layers of smooth muscle cells. There is a very thin layer of tunica intima lined by a layer of endothelial cells (arrows). The external elastic lamina is not distinct. The structure is quite similar to that seen in the human intracranial arteries except that the layer of the tunica media is slightly thicker in this model. B. Microscopic view of an artery treated with mechanical thrombectomy (H\&E staining). A low-magnification view (left, original magnification $\times 40$ ) shows the separation of the smooth muscle layers in the tunica media and migration of acute inflammatory cells between the layers (arrowheads). A magnified view of the same arterial sample (right, original magnification $\times 200$ ) demonstrates aggregated platelets on the surface of the arterial wall (arrows) and acute inflammatory cells migrating into the innermost layers of the tunica media (open arrows). The findings indicate an acute inflammatory reaction induced by the mechanical thrombectomy.

graphic outcome was 46.2 minutes. All of the embolized target vessels, which were surgically exposed, stopped pulsating and became pale/dusky immediately after vessel occlusion (Fig 2A, -B). The microcatheter and thrombectomy devices were observed, under direct visualization, through the transparent wall of the SCA (Fig 2C). The distal branches of the SCA were often significantly stretched during the deployment of the thrombectomy device.

\section{Harvesting and Histologic Evaluation of Treated Arteries}

A segment of the SCA, $5 \mathrm{~cm}$ in length, was surgically removed after the endovascular procedure. The harvested samples were photographed and sectioned transversely at 5-mm intervals from distal to proximal. Microscopic analysis of arterial samples showed a well-preserved layered structure of the vessel wall throughout the segment of each harvested artery (Fig 3A).

The SCAs that underwent mechanical thrombectomy demonstrated various histologic changes. Aggregated platelets on the surface of the arterial wall and separation of smooth muscle layers within the tunica media were seen. The invasion of acute inflammatory cells, most likely polymorphonuclear leukocytes, into the innermost layers of tunica media was also seen (Fig 3B). This finding indicated an acute inflammatory reaction induced by the mechanical thrombectomy. No large intimal flap or complete dissection of the tunica media was observed.

\section{DISCUSSION}

Human intracranial arteries are known to have thinner vessel walls than the small arteries in other parts of the human body. The mean arterial wall thickness of human cerebral arteries averages $94 \pm 30 \mathrm{im}$ with some minimal variation between the MCA and basilar and vertebral arteries, ${ }^{11}$ whereas the mean thickness of the swine SCA ranges between 120 and $200 \mu \mathrm{m}$. With that in mind, we need to be careful when applying observed vessel damage in this animal model to clinical scenarios involving human subjects. Secondarily, additional investigation with different harvesting time points is necessary to clarify whether the histologic changes observed in the current study evolve into chronic changes.

Real-time direct visualization of SCAs undergoing mechanical thrombectomy will contribute to our understanding of arterial structural changes induced by the mechanical thrombectomy devices, such as overstretching of the arterial wall, vasospasm, subadventitial hematoma, and so forth. The model also contributes to the understanding of procedure-related histologic changes induced in SCAs in swine and potentially by extrapolation in humans. A comparative analysis by using various types of thrombectomy devices can be performed by using this animal model. Further investigation with a larger number of specimens is required to better understand the cause-and-effect relationship between particular endovascular maneuvers and tissue reaction/damage observed in treated arteries.

Disclosures: Harry Vinters_UNRELATED: Stock/Stock Options: I own stock in (and receive dividends from) $3 \mathrm{M}$ (medical equipment manufacturer), Teva Pharma, Pfizer, and Glaxo Smith Kline Beecham.

\section{REFERENCES}

1. Gobin YP, Starkman S, Duckwiler GR, et al. MERCI 1: a phase 1 study of Mechanical Embolus Removal in Cerebral Ischemia. Stroke 2004; 35:2848-54

2. Smith WS, Sung G, Saver J, et al. Mechanical thrombectomy for acute ischemic stroke: final results of the Multi MERCI trial. Stroke 2008;39:1205-12

3. Shi ZS, Liebeskind DS, Loh Y, et al. Predictors of subarachnoid hemorrhage in acute ischemic stroke with endovascular therapy. Stroke 2010;41:2775-81

4. Getty R. The Anatomy of the Domestic Animals. Philadelphia: WB Saunders Company; 1975

AJNR Am J Neuroradiol 34:E87-E90 Aug 2013 www.ajnr.org 
5. Gomercic MD, Vukicevic TT, Galov TF, et al. The cisterna chyli and thoracic duct in pigs (Sus scrofa domestica). Vet Med (Praha) 2010; 55:31-34

6. Monson KL, Goldsmith W, Barbaro NM, et al. Significance of source and size in the mechanical response of human cerebral blood vessels. J Biomech 2005;38:737-44

7. Tanriover N, Kawashima M, Rhoton AL Jr, et al. Microsurgical anatomy of the early branches of the middle cerebral artery: morphometric analysis and classification with angiographic correlation. J Neurosurg 2003;98:1277-90

8. Gralla J, Schroth G, Remonda L, et al. A dedicated animal model for mechanical thrombectomy in acute stroke. AJNR Am J Neuroradiol 2006;27:1357-61

9. Kan I, Yuki I, Murayama Y, et al. A novel method of thrombus preparation for use in a swine model for evaluation of thrombectomy devices. AJNR Am J Neuroradiol 2010;31:1741-43

10. Gralla J, Schroth G, Remonda L, et al. Mechanical thrombectomy for acute ischemic stroke: thrombus-device interaction, efficiency, and complications in vivo. Stroke 2006;37:3019-24

11. Walmsley JG, Campling MR, Chertkow HM. Interrelationships among wall structure, smooth muscle orientation, and contraction in human major cerebral arteries. Stroke 1983;14:781-90 